What methods could be in the running for Method of the Year next year? Read on for a subjective selection of some of the possibilities.

\title{
》Targeted proteomics
}

Technology for sensitively and reproducibly detecting targeted proteins by mass spectrometry picks up speed.

In a typical 'shotgun' proteomic analysis, a mixture of proteins is digested into peptides, fractionated and analyzed by mass spectrometry. This in theory could lead to the discovery of all proteins in the sample, though in practice this is extremely challenging and requires huge resources. But for many types of experiments, the researcher does not necessarily want to discover new proteins but instead is interested in following what happens to a relatively small number of

Proteins can be detected with very high sensitivity and reproducibility through targeted proteomics approaches. proteins under different conditions.

For such experiments, targeted approaches such as selected reaction monitoring (SRM; or multiple reaction monitoring, MRM, as it is also commonly known) offer much higher sensitivity and much greater speed of analysis. Developing and validating SRM assays is an extensive process, but once generated for a particular protein, this mass spectrometric assay can be deployed for the accurate identification and quantification of that protein across biological samples.

Though the SRM approach has been around for many years, it has only recently been gaining traction in the field of proteomics. Ruedi Aebersold's group has been particularly influential in pushing the technology forward. This year, they showed that SRM could be used to detect expressed proteins over a wide dynamic range, down to the unprecedented level of fewer than 50 copies per cell (Cell 138, 795-806, 2009). In this issue, the Aebersold group also demonstrated that cheaply synthesized crude peptides can be used for

\section{\>ptical imaging of the native brain}

Methodological developments are opening the functioning brain to cellular-level investigation using light.

Despite extraordinary progress in figuring out how our organs provide the functions necessary for life, an in-depth understanding of how the brain works remains elusive. Unlike the heart or kidneys, brain structures we can easily discern provide few clues. Further complicating matters, the brain resides in a largely inaccessible skull.

Most investigation of the brain has involved examining the function of individual brain cells or examining the relationship between regional neuronal activity and organism function - studies dominated by electrophysiology and magnetic resonance imaging. Optical probes of activity provide a way of bridging these two scales of investigation, but until recently optical studies of the functioning intact brain were stymied by inadequate methods.

But new developments are redefining what is possible. Two-photon microscopy increases the penetration depth of light microscopy tenfold, making it possible to peer as much as a millimeter into the brain. Fluorescent sensors of neuronal activity are improving and report neuronal signaling with greater reliability. The coupling of these developments with preparative techniques that provide physical and optical the high-throughput generation of SRM assays (Nat. Methods 7, 43-43, 2010). Additionally, a multilaboratory study showed that SRM assays are transferrable and reproducible across laboratories (Nat. Biotechnol. 27, 633-641, 2009).

It will take a long time to build up a database of SRM assays for whole proteomes, but we are likely to see rapid growth in this area, which will facilitate high-throughput and highly sensitive protein detection for a wide variety of experiments.

Allison Doerr

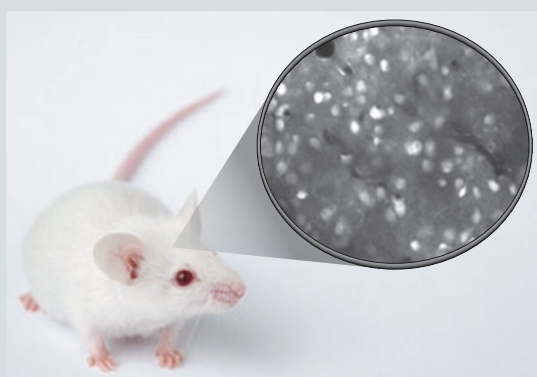

Methodological development in optical microscopy can now provide cellular-level information on neuronal signaling in behaving mice and rats.

access to the brain of mice and rats has made it possible to visualize neuronal signaling in the intact brain of living animals.

Early studies were limited to anesthetized mice, which limited the applications. Neuroscientists now have mice that walk on spinning Styrofoam balls in virtualreality environments while a two-photon microscope records the firing of networks of neurons (Nature 461, 941-946, 2009). Miniaturized two-photon microscopes can be carried by freely moving rats while imaging takes place (Proc. Natl. Acad. Sci. USA 106, 19557-19562, 2009). Or fiberbased microscopes can peer into the deep recesses of the brain in freely moving mice (Nat. Methods 5, 935-938, 2008).

The spatial scales covered by these new methods-from subcellular to networks of hundreds of cells-promise to open new doors of understanding for this most impenetrable of organs. Daniel Evanko 